Conclusion This research identifies key risk factors pertaining to female drowning and contributes to SDGs 3 good health and wellbeing and 5 gender equality. It has the potential to inform future drowning prevention interventions for females and the development of future research into the drivers of female drowning, both within New Zealand and globally.

\section{B.005 DYING TO HELP: FATAL BYSTANDER RESCUES IN AUSTRALIAN COASTAL ENVIRONMENTS}

${ }^{1} J a z$ Lawes*, ${ }^{2}$ Rob Brander, ${ }^{3}$ Richard Franklin, 'Shane Daw. 'Surf Life Saving Australia, Sydney, Australia; ${ }^{2}$ UNSW, Sydney, Australia; ${ }^{3}$ James Cook University, Townsvile, Australia

10.1136/injuryprev-2021-safety.41

Bystanders who drown attempting a rescue are becoming an increasingly important issue within drowning prevention. In Australia, most of these incidents occur in coastal waterways. This study characterises coastal bystander rescuer fatalities collated in the national coastal fatality database (2004-2019) to guide future public safety interventions involving bystander rescuers. Sixty-seven bystander rescuer fatalities in coastal waterways were reported during the 15 -year period, an average of 4.5 per year, which is a significant proportion of the five fatalities previously reported across all Australian waterways.

Most coastal bystander rescuer fatality incidents occurred in New South Wales (49\%), at beaches (64\%), in regional or remote areas $(71 \%)$, more than $1 \mathrm{~km}$ from the nearest lifesaving service (78\%), during summer $(45 \%)$, in the afternoon (72\%), in the presence of rip currents (73\%), and did not involve the use of flotation devices to assist rescue (97\%). The majority of coastal bystander rescuer victims were Australian residents (88\%) born in Australia/Oceania (68\%), males (81\%), aged between 30-44 years old $(36 \%)$, were visitors to the location $(55 \%)$, either family $(69 \%)$ or friends $(15 \%)$ of the rescuee(s), and were attempting to rescue someone younger than 18 years old (64\%).

Our results suggest safety intervention approaches should target males, parents and carers visiting beach locations in regional locations and should focus on the importance of flotation devices when enacting a rescue and further educating visitors about the rip current hazard. Future research should examine the psychology of bystander rescue situations and evaluate the effectiveness of different safety intervention approaches.

\section{C - Falls, March 23, 2021}

\section{C.001 TRENDS IN FALLS-RELATED INJURY HOSPITALISATIONS AND DEATHS AMONG ADULTS IN VICTORIA, AUSTRALIA}

\footnotetext{
1,2Aleksandra Natora*, 'Jennie Oxley, ${ }^{1}$ Terry Haines, 'Linda Barclay, ${ }^{2}$ Bruce Bolam. ${ }^{1}$ Monash University, Melbourne, Australia; ${ }^{2}$ Department of Health and Human Services Victoria, Melbourne, Australia
}

10.1136/injuryprev-2021-safety.42
Background In the Australian state of Victoria, rates of unintentional falls-related deaths and hospitalisations among community-dwelling older people living in their own homes are increasing. Despite the availability of evidence-based falls prevention interventions the rates are projected to keep rising with the ageing of the population.

Methods Retrospective quantitative data analysis was conducted of falls-related injury hospitalisations and deaths among community-dwelling older people in Victoria, for the period 2006 to 2018, captured by key datasets on deaths and hospitalisations available in Victoria from the Australian Bureau of Statistics (ABS), National Coronial Information System (NCIS) and the Victorian Agency for Health Information (VAHI). Data analysis includes temporal trends and descriptive statistics.

Results This presentation will share results of the data analysis. Highlights will include temporal trends in fall-related deaths and hospitalisations, stratified by age and sex, fall type, injury type and activity prior to fall-related injury.

Conclusions The burden of high and low falls across the adult life-course warrants increased government policy and investment in primary prevention of falls, and for efforts to begin earlier than at $65+$ years of age.

Learning outcomes This presentation of the latest surveillance of the Victorian population falls-related injury will inform future government policy effort in the primary prevention of falls in the community. Information about the Victorian population may have relevance to other international ageing populations.

\section{C.002 GRAVITY CAN BE A REAL KILLJOY. FALL ACCIDENTS INVOLVING 0-4-YEAR OLD'S IN RESIDENCES}

${ }^{1}$ Peter Spitzer* ${ }^{*}{ }^{1,2}$ Holger Till. ${ }^{1}$ Research Center for Childhood Injuries at Safe Kids Austria, Graz, Austria; ${ }^{2}$ Medical University, Graz, Austria

10.1136/injuryprev-2021-safety.43

Background Fall accidents are responsible for about a quarter of all injuries and hospital treatments in Austria. Children in the age group of 0-4-year-olds undergo hospital medical examinations even more frequently due to accidents resulting from falls.

Methods An analysis was conducted of fall accidents suffered by $0-4$-year-olds in residences, who were treated at the Department for Paediatric and Youth Surgery, by using the injury data base.

Results 4,709 accidents were identified as 'falls at home'. In this group of 0 -4-year-olds a peak could be observed during the first year of life, during which $28 \%$ of the falls occurred.

In the case of injuries, $18 \%$ were categorized as being medically serious injuries.

Thirty-nine percent of the falls could be categorized as falls from height. The couch in the living room was the most common piece of furniture from which falls occurred.

Conclusion In Austria, safety standards and laws are central components of human protection, which is why technical prevention will no longer be the central starting point for accident prevention work. In many cases, the behaviour of a child who is subject to psychomotor developmental conditions and/or the behaviour of an adult/parent is the effective starting point for safety work. 